\title{
On the space of left-orderings of virtually solvable groups
}

\author{
Cristobal Rivas*\& Romain Tessera ${ }^{\dagger}$
}

\begin{abstract}
We show that the space of left-orderings of a countable virtually solvable group is either finite or homeomorphic to a Cantor set. We also provide an explicit description of the space of left-orderings of $S O L=\mathbb{Z}^{2} \rtimes_{T} \mathbb{Z}$.
\end{abstract}

\section{Introduction}

The space of left-orderings $\mathcal{L O}(G)$ of a left-orderable group $G$ is the set of all possible left-orderings on $G$ endowed with a natural topology that makes it compact, Hausdorff and totally disconnected, see [25] or 2.1, It was proved by Linnell that this space is either finite or uncountable [11. The problem of relating the topology of $\mathcal{L O}(G)$ with the algebraic structure of $G$ has been of increasing interest since the discovery by Dubrovina and Dubrovin that the space of left-orderings of the braid groups is infinite and yet contains isolated points [6]. Recently, more examples of groups showing these two behaviors have appeared in the literature [4, 8, 9, 16]. All these groups admit a nonabelian free subgroup. However, non-abelian free groups [12], and more generally non-trivial free products of groups have no isolated left-orderings [23].

In any case, the class of groups having isolated orderings is far from being well understood. By contrast, left-orderable groups admitting only finitely many left-orderings have been classified by Tararin [10, Theorem 5.2.1]. For short, we shall call these groups "Tararin groups". Tararin groups form a very restrictive and easy to describe class of finite-rank-solvable1 1 groups, see $\$ 2.2$. As already mentioned, these are the only known examples of left-orderable amenable groups with isolated points. Could they be the only ones? This paper brings in a modest contribution to this problem, answering it positively for (virtually) solvable groups.

Theorem 1.1. The space of left-orderings of a countable virtually solvable group is either finite or a Cantor set.

In particular we deduce

Corollary 1.2. If $\Gamma$ is a (countable) left-orderable virtually solvable group of infinite rank, then $\mathcal{L O}(\Gamma)$ is a Cantor set.

Among Tararin groups, those which are virtually polycyclid turn out to be virtually nilpotent, therefore we deduce

\footnotetext{
${ }^{*}$ The first author was supported by CONICYT and labex MILYON.

${ }^{\dagger}$ The second author was supported by ANR BLAN AGORA and ANR BLAN GGAA.

${ }^{1} \mathrm{~A}$ solvable group has finite rank, if in its derived series all successive quotients have finite rank as Abelian groups, see $[24$.

${ }^{2} \mathrm{~A}$ polycyclic group, is a solvable group whose successive Abelian quotients, in its derived series, are finitely generated, see [20].
} 
Corollary 1.3. If $\Gamma$ is a left-orderable virtually polycyclic group of exponential growth, then $\mathcal{L O}(\Gamma)$ is a Cantor set.

The study of left-orderable amenable groups is intimately related to that of Conradian orderings (see 2.5 for a definition) and to local indicability. Recall that Morris gave a beautiful proof of the fact that all left-orderable amenable groups admit Conradian orderings [13] (see also [14, 2] for older results in that direction, and [5] for an interesting alternative proof). Together with a fundamental observation of Conrad [3, this provides a very natural characterization of left-orderable amenable groups as those which are locally indicable, i.e. all their finitely generated subgroups have a nontrivial morphism to $\mathbb{Z}$.

The dichotomy shown in Theorem 1.1 reminds of a similar one, this time for all groups but in restriction to Conradian orderings. Indeed, in [21] the first author proved that the space of Conradian orderings of a countable group is either finite or homeomorphic to the Cantor set. This implies for instance, the general dichotomy for left-orderable groups having only Conradian orderings, such as groups of sub-exponential growth [15].

\section{Remarks about the proof}

It is now well known that countable groups are left-orderable if and only if they act faithfully by order-preserving homeomorphisms on the real line (see for instance [7] or 92.3 ). This dynamical approach have shown to be fruitful when trying to understand the topology of the space of leftorderings of a given group or family of groups, see for instance [15, 21, 23]. In this work, this point of view will be crucial.

In a previous version of this paper, Theorem 1.1 was proved for the class of finite-rank solvable groups. An important feature of this subclass is that they are virtually nilpotent-by-Abelian [24]. In this case, the proof can be summarized as follows: if the ordering is not Conradian (in which case, the conclusion follows from [15]), then one can prove that the ordering is induced, up to semiconjugacy, from an affine action on the real line. It is then not to hard to see that the underlying ordering is non-isolated, see Proposition 2.12 and Corollary 2.13. This approach strongly relies on the work of Plante [19], who shows that any action of a finite-rank solvable group on the real line quasi preserves a Radon measure. However, as already noted by Plante, there are actions of solvable groups on the real line (such as $\mathbb{Z} \imath \mathbb{Z}$ ) in which no non-trivial Radon measure is quasi-preserved. This situation is much more subtle and requires a careful dynamical analysis. Roughly speaking, the proof consists in showing that the action behaves "at a certain scale" like an affine action (see Section 4 and Lemma (5.6).

\section{Organization of the paper}

In $₫ 2$ we give some necessary background. Most of the material is well known, but not all. Notably Corollary 2.16 plays a crucial role in our proof. In $\$ 3$ we prove Theorem 1.1 in a simple example 3 , namely $S O L$. We also give an explicit description of its space of left-orderings. In $\$$ we illustrate the difficulties arising when dealing with solvable groups of infinite rank on a specific example, due to Plante, of an action of $\mathbb{Z} \imath \mathbb{Z}$ on the line. Finally the proof of Theorem 1.1 is carried out in 95 .

Remark 1.4. In various places we employ the useful terminology "pseudo-ordering" (Definition 2.4) which means an invariant ordering on a quotient of $G$ by a (not necessarily normal) subgroup, but seen as a partial ordering on $G$.

\footnotetext{
${ }^{3}$ Solvable Baumslag-Solitar groups could have been treated in a similar way, we leave the easy adaptation of the proof to the reader.
} 
Acknowledgements: The first-named author would like to thanks the hospitality of ENS-Lyon where he enjoys a one year and a half posdoctoral position. He is specially grateful to M. Triestino, A. Glutsyuk and É. Ghys for interesting discussions around this and other related problems.

\section{Preliminaries}

\subsection{The topology on $\mathcal{L O}(G)$}

A basis of neighborhoods in $\mathcal{L O}(G)$ is the family of the sets $V_{f_{1}, \ldots, f_{k}}:=\left\{\preceq \mid i d \preceq f_{1}, \ldots, i d \preceq\right.$ $\left.f_{k}\right\}$, where $\left\{f_{1}, \ldots, f_{k}\right\}$ runs over all finite subsets of $G$. If $G$ is countable, then this topology is metrizable. For instance, if $G$ is finitely generated, we may define $\operatorname{dist}\left(\preceq, \preceq^{\prime}\right)=1 / 2^{n}$, where $n$ is the first integer such that $\preceq$ and $\preceq^{\prime}$ do not coincide on $n$-th ball (with respect to some generator system). For each $g \in G$ and $\preceq \in \mathcal{L O}(G)$, one can define an other element $g(\preceq) \in \mathcal{L O}(G)$ whose positive cone is the set of elements $f \in G$ such that $g f g^{-1} \succ i d$. This defines a continuous representation of $G$ in $H$ omeo $(\mathcal{L O}(G))$ called action by conjugation of $G$ on its space of leftorderings.

The following definition is classical [1, 10]. Given a left-ordered group $(G, \preceq)$ and a subgroup $H$, we say that $H$ is convex if for every $g \in G$ such that $h_{1} \preceq g \preceq h_{2}$, for $h_{1}, h_{2}$ in $H$, we have that $g \in H$. Convex subgroups have the nice property that they induce a total ordering on the left-cosets $G / H$ by

$$
g_{1} H \preceq^{*} g_{2} H \Leftrightarrow g_{1} h_{1} \preceq g_{2} h_{2} \text { for all } h_{1}, h_{2} \text { in } H .
$$

This ordering is invariant under the $G$-action by left translation on $G / H$. In particular, if $H$ is normal, then $G / H$ is a left-orderable group, in which case we call $\swarrow^{*}$ the projected or quotient ordering. It follows that $\preceq$ decomposes "lexicographically" as the ordering on the $H$-cosets and the ordering restricted to $H$. More precisely we have that

$$
i d \prec g \Leftrightarrow\left\{\begin{array}{c}
H \prec \prec^{*} g H, \text { or } \\
H=g H \text { and } i d \prec g
\end{array}\right.
$$

Elaborating on this, we conclude (see [22] for more details)

Proposition 2.1. Let $\preceq$ be a left-ordering on $G$ and let $H$ be a convex subgroup. Then there is a continuous injection $\mathcal{L O}(H) \rightarrow \mathcal{L O}(G)$, having $\preceq$ in its image. Moreover, if in addition $H$ is normal, then there is a continuous injection $\mathcal{L O}(H) \times \mathcal{L O}(G / H) \rightarrow \mathcal{L O}(G)$ having $\preceq$ in its image.

Remark 2.2. Let $\preceq$ be a left-ordering on $G$, and $H$ a normal convex subgroup. Then, it is not hard to check that if the restriction of $\preceq$ to $H$ and the projection of $\preceq$ to $G / H$ are Conradian, then $\preceq$ is also Conradian.

Observe that the set of convex subgroups of a given left-ordering, are totally ordered for the inclusion. We call it the convex series of $G$. The following Corollary is well known, and appears for instance in [21]. For the readers' convenience we sketch a proof. Let $G$ be a group and $H$ be a (not necessarily normal) subgroup. Then to any $G$-invariant ordering $\preceq$ on $G / H$, one can define the so-called opposite ordering $\preceq^{o p}$ defined by $g \prec^{o p} f \Leftrightarrow g \succ f$. We sometimes say that $\preceq^{o p}$ is obtained by flipping $\preceq$. Clearly $\preceq_{o p}^{o p}$ is also $G$-invariant.

Corollary 2.3. Suppose that a left-ordered group $(G, \preceq)$ has infinitely many convex subgroup. Then $\preceq$ is non-isolated. 
Sketch of the proof: If the convex series is infinite, then either there exists an infinite increasing sequence of convex subgroups $C_{1}<C_{2} \ldots$ or an infinite decreasing sequence $C_{1}^{\prime}>C_{2}^{\prime} \ldots$ Flipping the ordering on $C_{n+1} / C_{n}$ (resp. on $C_{n}^{\prime}$ ), one obtains a sequence of orderings $\preceq_{n}$, distinct from $\preceq$, which converges to $\preceq$ when $n$ goes to infinity.

Note that we often have to deal with orderings on a quotient $G / H$, where $H$ is not necessarily normal. It will therefore be convenient to see $G$-invariant orderings on $G / H$ as a "pseudo-ordering" on $G$, namely

Definition 2.4. (Pseudo-ordering) A pseudo-ordering $\preceq$ on a group $G$ is a left-invariant partial ordering on $G$ induced from a $G$-invariant ordering $\preceq^{*}$ on a quotient $G / H$ : $i d \prec g \Leftrightarrow H \prec^{*} g H$. Given a pseudo-ordering on $G$, the set of elements which are not comparable to the neutral element coincides with the subgroup $H$.

- (Convex subgroup) A convex subgroup $C$ of a pseudo-ordered group is defined similarly as for orderings, with the additional requirement that $C$ must contain $H$. Note that $H$ itself is convex, and so is the minimal convex subgroup.

- (Quotient by a convex subgroup) Given a pseudo-ordering $\preceq$ on $G$ and $C$ a convex subgroup, the pseudo-ordering on $G$ induced from the ordering on $G / C$ will be called the quotient of $\preceq$ by $C$.

Remark 2.5. If $\preceq^{\prime}$ is the quotient of $\preceq$ by a convex subgroup $C$ of $(G, \preceq)$, then convex subgroups of $\left(G, \preceq^{\prime}\right)$ are exactly those convex subgroups of $(G, \preceq)$ containing $C$.

\subsection{Tararin groups}

We give a slight modification of the original statement of Tararin [10, Theorem 5.2.1], describing groups admitting only finitely many left-orderings. Recall that a series

$$
\{1\}=G_{0} \triangleleft G_{1} \triangleleft \ldots \triangleleft G_{m}=G,
$$

is said to be rational if each quotient $G_{i+1} / G_{i}$ is torsion-free rank-one Abelian.

Theorem 2.6 (Tararin). Let $G$ be a left-orderable group. If $G$ admits only finitely many leftorderings, then $G$ admits a unique (hence characteristic) rational series

$$
\{1\}=G_{0} \triangleleft G_{1} \triangleleft \ldots \triangleleft G_{m}=G,
$$

such that, for every $2 \leq i \leq m$, there is an element of $G_{i} / G_{i-1}$ whose action by conjugation on $G_{i-1} / G_{i-2}$ is by multiplication by a negative rational number. We shall call such a group a Tararin group.

Remark 2.7. The left-orderings on a Tararin group $G$ are very easy to describe. Indeed, if $\{1\}=G_{0} \triangleleft \ldots \triangleleft G_{m}=G$, is the associated rational series, then on each quotient $G_{i} / G_{i-1}$, being rank-one torsion free Abelian, there is -up to flipping- a unique left-ordering coming from an embedding into $\mathbb{Q}$. For every $i$, let $\preceq_{i}$ be a choice of an ordering on $G_{i} / G_{i-1}$. Then we can produce a left-orderings on $G$ by declaring

$$
g \succ i d \Leftrightarrow\left\{\begin{array}{c}
g G_{m-1} \succ_{m} G_{m-1}, \text { or } \\
g \in G_{m-1}, \text { and } g \succ_{m-1} i d, \text { or } \\
\vdots \\
g \in G_{1}, \text { and } g \succ_{1} i d .
\end{array}\right.
$$


It is not hard to check that in this way we can produce all possible $\left(2^{m}\right)$ left-orderings (in fact, it is easy to show that they are all Conradian). Moreover, in any such ordering, the groups $G_{i}$ are convex, and conversely, every convex subgroup is of this form.

Corollary 2.8. Let $G$ be a virtually polycyclic group admitting only finitely many left-orderings. Then it admits a unique filtration such that $G_{i} / G_{i-1} \simeq \mathbb{Z}$. The action of (the generator of) $G_{i} / G_{i-1}$ on $G_{i-1} / G_{i-2}$ is by multiplication by -1 .

Since in a virtually polycyclic group, the group generated by $\left\{g^{2} \mid g \in G\right\}$ has finite index [20], we deduce

Corollary 2.9. A virtually polycyclic group having only finitely many left-orderings is virtually nilpotent.

We finish this section with a rigidity statement for actions of Tararin groups on the line. Let $G$ be a Tararin group and $\{1\}=G_{0} \triangleleft G_{1} \triangleleft \ldots \triangleleft G_{m}=G$ its associated rational series. Since $G_{m-1}$ is convex in every left-ordering of $G$, and $G / G_{m-1}$ is Abelian we have that the sign of $\gamma \in G \backslash G_{m-1}$ is preserved under conjugation. On the other hand, Remark 2.7 says that starting from a leftordering $\preceq$, any other left-ordering on $G$ is obtained by flipping the ordering on some of the convex subgroups. It follows from Theorem 2.6, that any flipping on any proper convex subgroup can be realized as conjugations by some element in $G$. This shows

Proposition 2.10. The conjugation action of a Tararin group $G$ on $\mathcal{L O}(G)$ has two orbits. Moreover, for any pair of left-orderings $\preceq, \preceq^{\prime}$ of $G$ there is $g \in G$ such that $g(\preceq)$ and $\preceq^{\prime}$ coincide over $G_{m-1}$. Moreover, if we let $\gamma_{T}$ be an element in $G \backslash G_{m-1}$ which acts on $G_{m-1} / G_{m-2}$ as multiplication by a negative number, then $g$ can be taken either in $G_{m-1}$ or in $\gamma_{T} G_{m-1}$.

\subsection{Dynamical realization}

As mentioned in the introduction, an important ingredient in our proof of Theorem 1.1 is the fact that countable left-orderable groups naturally act by order-preserving automorphism of the real line, and vice versa, a group acting faithfully by order-preserving automorphism of the real line is left-orderable [7].

More precisely, given a left-ordered group $(G, \preceq)$, there is an embedding of $G$ into Homeo+ $(\mathbb{R})$, the group of order preserving automorphism of the real line, such that:

- $G$ acts without global fixed points,

- for $f, g$ in $G$, we have that $f \prec g \Leftrightarrow f(0)<g(0)$, and

- the set of fixed points of a non-trivial $f \in G$ has empty interior.

This construction extends to pseudo-orderings in the sense that every pseudo-order on $G$ with minimal convex subgroup $H$ can be induced from an action of $G$ on the real line, where $H$ is the stabilizer of 0 .

We call such an action, a dynamical realization of $(G, \preceq)$. Conversely, given an embedding of $G$ into $\mathrm{Homeo}_{+}(\mathbb{R})$, we can induce a left-ordering as follows: take $\left(x_{1}, x_{2}, \ldots\right)$ a dense sequence in $\mathbb{R}$, and declare that an element $g \succ_{\left(x_{1}, x_{2}, \ldots\right)} i d$ if and only if $g\left(x_{i}\right)>x_{i}$, where $i$ is such that $g\left(x_{j}\right)=x_{j}$ for every $j<i$. We call such an ordering, an induced ordering from the action. Note that with this procedure we can recover a left-ordering from its dynamical realization by taking $x_{1}=0$.

Remark 2.11. Let $g \in G$, and $\preceq=\preceq\left(x_{1}, x_{2}, \ldots\right)$ be an ordering induced from an action of $G$ on the real line. Then $g(\preceq)$ is the left-ordering induced from the sequence $\left(g^{-1}\left(x_{1}\right), g^{-1}\left(x_{2}\right), \ldots\right)$. 


\subsection{Orderings induced by affine actions}

A general procedure for trying to approximate a given left-ordering $\preceq$ on a countable group, is to consider its dynamical realization, and to induce an ordering $\preceq^{\prime}$ from a sequence $\left(x_{1}, x_{2}, \ldots\right)$ where $x_{1}$ is close to 0 . The fact that $\preceq^{\prime}$ is close to $\preceq$ when $x_{1}$ is close to 0 follows from the continuity of the action, and from the fact that 0 has a free orbit (details are left to the reader). The problem however, is that the two orbits may induce the same ordering. This is the case for instance if $\preceq$ has a least positive element.

Our first step in proving Theorem 1.1 is that left-orderings induced from non-Abelian affine actions, are non-isolated.

Proposition 2.12. Let $\Gamma$ be a countable group, and suppose a left-ordering $\preceq$ on $\Gamma$ is induced from a faithful (order-preserving) affine action on the real line. Then, if $\Gamma$ is non-Abelian, $\preceq$ is approximated by its conjugates.

Proof: Let $\preceq=\preceq_{\left(x_{1}, x_{2}, \ldots\right)}$ be the left-ordering induced from the sequence $\left(x_{1}, x_{2}, \ldots\right)$. We note that, since the elements in the affine group have at most one fixed point, it is enough to specify two points. So $\preceq=\preceq\left(x_{1}, x_{2}\right)$.

By assumption, $\Gamma$ has both non-trivial homotheties and non-trivial translations. It follows that the subgroup made of translations has dense orbits. In particular, the countable set $\Omega$ consisting of the points in $\mathbb{R}$ which are fixed by some non-trivial element (homothety) of $\Gamma$, is also dense in $\mathbb{R}$. Therefore, given any two points $x_{1}, y_{1} \in \mathbb{R}, x_{1} \neq y_{1}$, there is a non-trivial homothety $h \in \Gamma$, having its unique fixed point between $x_{1}$ and $y_{1}$. In particular, the left-orderings $\preceq_{\left(x_{1}, x_{2}\right)}$ and $\preceq_{\left(y_{1}, y_{2}\right)}$, induced from $\left(x_{1}, x_{2}\right)$ and $\left(y_{1}, y_{2}\right)$ respectively, are different.

We now show that $y_{1}$ may be chosen so that $\preceq_{\left(y_{1}, y_{2}\right)}$ is close to $\preceq_{\left(x_{1}, x_{2}\right)}$. As noted earlier, this is obvious if $x_{1}$ has a free orbit, so let us suppose that it is not the case. Let $\operatorname{Stab}_{\Gamma}\left(x_{1}\right)$ be the stabilizer of $x_{1}$ in $\Gamma$, and let $S \subset \Gamma$ be a finite set of $\preceq$-positive elements. We write $S=S_{1} \cup S_{2}$ where $S_{1}=S \cap \operatorname{Stab}_{\Gamma}\left(x_{1}\right)$, and we call $I$ the open interval between $x_{1}$ and $x_{2}$. Since $S_{2}$ is finite, there is a small neighborhood $U$ of $x_{1}$ such that $\gamma(x)>x$ for every $x \in U$ and every $\gamma \in S_{2}$. On the other hand, for every $\gamma \in S_{1}$, we have that $\gamma(x)>x$ for every $x \in I$ (recall that $\gamma \in S_{1}$ is an homothety). Thus, if we take $y_{1} \in I \cap U$ then $\preceq_{\left(y_{1}, y_{2}\right)}$ and $\preceq\left(y_{2}\right.$ being any point) are in the same open set associated to $S$. Since $I \cap U$ has non-empty interior, it is easy to see that $\left(y_{1}, y_{2}\right)$ may be chosen so that $\left(y_{1}, y_{2}\right)=\left(\gamma\left(x_{1}\right), \gamma\left(x_{2}\right)\right)$ for some $\gamma \in \Gamma$, which shows that $\preceq$ is approximated by its conjugates.

To state the following corollary, recall that given two actions $A_{1}, A_{2}$ of a group $\Gamma$ on the real line, we say that $A_{1}$ is semi-conjugated to $A_{2}$ if there is an increasing surjective function $F: \mathbb{R} \rightarrow \mathbb{R}$ such that

$$
F \circ A_{1}(\gamma)=A_{2}(\gamma) \circ F(\forall \gamma \in \Gamma) .
$$

Corollary 2.13. Let $(\Gamma, \preceq)$ be a countable, left-ordered group. Suppose there is an affine (orderpreserving) action $A: \Gamma \rightarrow A f f_{+}(\mathbb{R})$ whose kernel is convex in $\preceq$, and whose range is non-Abelian. Suppose further that the dynamical realization of $(\Gamma, \preceq)$ is semi-conjugated to $A$. Then $\preceq$ is nonisolated.

Proof: In light of Proposition 2.1, it is enough to show the corollary when $A$ is a faithful action. Let $F: \mathbb{R} \rightarrow \mathbb{R}$ be the function that realizes the semi-conjugation, and let $\operatorname{Stab}_{A(\Gamma)}(F(0))$ be the stabilizer of $F(0)$ in $A(\Gamma)$ : this is an Abelian subgroup of $\Gamma$. We claim that it is convex. Indeed, if $\gamma_{1} \prec g \prec \gamma_{2}$, then $\gamma_{1}(0)<g(0)<\gamma_{2}(0)$. So $F\left(\gamma_{1}(0)\right) \leq F(g(0)) \leq F\left(\gamma_{2}(0)\right)$, and hence $A\left(\gamma_{1}\right)(F(0)) \leq A(g)(F(0)) \leq A\left(\gamma_{2}\right)(F(0))$. From where the claim follows. 
Now, if $\operatorname{Stab}_{A(\Gamma)}(F(0))$ is trivial then Proposition 2.12 applies directly, since in this case $\preceq$ is realized as the induced ordering from $F(0)$ in the action $A$. If it has rank $>1$, then the restriction of $\preceq$ to $\operatorname{Stab}_{A(\Gamma)}(F(0))$ is non-isolated, thus $\preceq$ itself is non-isolated.

Now, if $\operatorname{Stab}_{A(\Gamma)}(F(0))$ has rank exactly 1, then the order restricted to $\operatorname{Stab}_{A(\Gamma)}(F(0))$ is completely determined by the sign of any given non-trivial element, say $A(t) \in \operatorname{Stab}_{A(\Gamma)}(F(0))$. Assume $t \succ 0$, then, because $A(t)$ acts as a non-trivial homothety, there exists $x \in \mathbb{R}$ such that $A(t)(x)>x$. It follows that $\preceq$ coincides with $\preceq_{\left(x_{1}, x_{2}\right)}$, the ordering induced form the action $A$ where $x_{1}=F(0)$ and $x_{2}=x$. So $\preceq$ is non-isolated by Proposition 2.12 .

\subsection{Conradian orderings}

There is a special type of left-ordering, introduced in [3], which will be very important in our proof of Theorem 1.1. These are the so called Conradian orderings, which are left-orderings satisfying the following additional property 4 :

$$
f \succ i d, \quad g \succ i d \Rightarrow f g^{2} \succ g .
$$

It turns out that Conradian orderings have a very interesting dynamical counterpart. Recall from [15], that $f, g \in \mathrm{Homeo}_{+}(\mathbb{R})$ are said to be crossed, if one of them, say $g$, has a domain $I$ (that is, an open interval, not necessarily bounded, which is fixed by $g$, and on which $g$ acts without fixed points), such that $f(I)$ is not equal, nor disjoint to $I$. A group $G \subset \mathrm{Homeo}_{+}(\mathbb{R})$ is said to act without crossings, if it does not contains crossed elements.
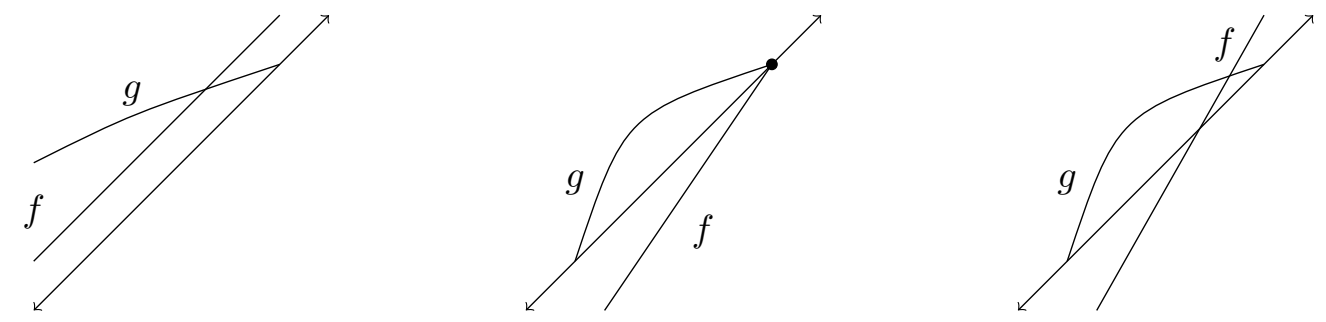

Figure 1: Three different crossings

Theorem 2.14 (Navas [15]). The dynamical realization of a Conradian ordering on $G$ is an action without crossings. Conversely, an induced ordering from an action without crossings is Conradian.

The above theorem implies rather easily that, in a dynamical realization of a Conradian ordering, the set of elements having fixed point is a subgroup (obviously normal). With this, together with a theorem of Hölder stating that every group acting freely on the real line is Abelian (see for instance [7]), one can deduce (see for instance [15]. Compare with [3].)

Corollary 2.15. Let $G$ be a countable group, $\preceq$ be a Conradian ordering of $G$, and $N \subset G$ be the set of elements having a fixed point in the dynamical realization of $(G, \preceq)$. Then $N$ is a normal subgroup of $G$. Moreover, if there is $g \in G$ having no fixed point (for instance if $G$ is finitely generated), then $G / N$ is a non-trivial torsion-free Abelian group which acts freely on the (non-empty) set of global fixed points of $N$.

Let us state here a last corollary which plays a crucial role in the proof of Theorem 1.1. It describes some constrains on the dynamics of a group acting on the real line, when it has a normal subgroup acting without crossings. More precisely

\footnotetext{
${ }^{4}$ In fact, in [3] the required property is $f \succ i d, g \succ i d \Rightarrow \exists n \geq 1$ such that $f g^{n} \succ g$. The fact that $n=2$ is enough is a result from Navas and Jiménez, see [15].
} 
Corollary 2.16. Let $(G, \preceq)$ be a left-ordered group, let $H$ be a normal subgroup such that $(H, \preceq)$ is Conradian, and consider a dynamical realization of $(G, \preceq)$. Let $f \in G, g \in H$ and let $I$ be $a$ minimal open interval fixed by $\mathrm{g}$. Then one of the following holds.

- $f(I)=I$ or

- $f(I)$ is disjoint from $I$, or

- (up to changing $f$ by its inverse) $\bar{I} \subset f(I)$. In this last case we say that $f$ acts as a dilation on $I$.

Proof: Notice that $f(I)$ is a domain of $g^{f}=f g f^{-1} \in H$. Hence, it follows from Theorem 2.14 that $g$ and $g^{f}$ are not crossed. In particular, $I$ and its image by $f$ are either disjoint or one is contained in the other. Indeed, if this is not the case, then $f(I)$ would not be fixed, nor moved disjointly by $g$. So, up to changing $f$ by $f^{-1}$, we can assume that $I \subseteq f(I)$.

We still have to rule out the possibility that these two intervals, although different, share a common extremity. This is again easy. Indeed, suppose it is the case that $I$ and $f(I)$ share a common extremity but $I \subsetneq f(I)$. Then $g^{f}$ can not move $I$ disjoint from itself. But, on the other hand, since $g^{f}$ have no fixed points inside $f(I)$, we have that $I$ can not be fixed by $g^{f}$, contradicting Theorem 2.14.

\section{The space of orderings of $S O L$}

\subsection{The space of orderings of $S O L$ is a Cantor set}

In this section we treat the simplest case of a non-virtually nilpotent polycyclic group, namely the group $S O L=\mathbb{Z}^{2} \rtimes_{T} \mathbb{Z}$, where $T$ is an hyperbolic matrix (that is, a matrix in $S L_{2}(\mathbb{Z}$ ) having trace greater than 2). In particular, $T$ has two irrational eigenvalues. We begin by proving that this group has no isolated orderings, and then we provide a quite explicit description of its set of orderings (describing for instance its bi-invariants orderings, which are left-orderings whose positive cone is preserved under conjugation).

We denote by $H$ the derived subgroup of $S O L$, which is isomorphic to $\mathbb{Z}^{2}$, and by $t$ the element of $\mathbb{Z}$ acting on $H$ as $T$. Let $\preceq$ be a left-ordering on $S O L$, and consider its dynamical realization.

Since $H$ is Abelian, the set of elements in $H$ acting with fixed points form a subgroup $H^{\prime}$. This subgroup, being finitely generated, actually has a global fixed point. But because $T$ is $\mathbb{Q}$ irreducible, this subgroup is either trivial or must have finite index. In the latter situation, we have that $H^{\prime}=H$ as every global fixed point of $H^{\prime}$ has a finite $H$-orbit, so must be fixed by $H$. We therefore have two cases to consider, namely

- Case 1. H has a global fixed point.

Let $I$ be the maximal open interval around 0 without global fixed point of $H$. Since $H$ is normal in $S O L$, and $S O L$ acts without global fixed points, we have that the set of global fixed points of $H$ is permuted by $S O L$ and therefore must be infinite. In particular, $I$ is a bounded interval which is either fixed or moved disjointly from itself by the action of $S O L$. Therefore $H=\operatorname{Stab}(I)$ is convex. Moreover, since $H$ has rank two, the restriction of $\preceq$ to $H$ is non-isolated. Hence, Proposition 2.1 implies that $\preceq$ itself is non-isolated. Observe moreover that $\preceq$ is Conradian.

- Case 2. H has no global fixed point. 
It follows that $H$ acts freely on the real line, and so, by Hölder's Theorem [7], it is semiconjugated to a group of translation. Now recall that Lebesgue's measure is the unique measure, up to scalar multiple, preserved by $\mathbb{Z}^{2}$ acting faithfully by translations. In particular $H$ preserves a unique atomless Radon 5 measure $\mu$. Moreover, the hyperbolicity of $T$ implies that $t$ does not preserves the measure $\mu$, but it acts on it as a dilation by one of the two eigenvalues of $T$. We therefore obtain a faithful embedding of $S O L$ in $A f f_{+}(\mathbb{R})$ which is realized by a semi-conjugation (see equation (11) and (2) respectively). We then conclude from Corollary 2.13. We observe that in this case, the proof of Corollary 2.13 shows that the ordering $\preceq$ is realized as an induced ordering from the associated affine action.

\subsection{Description of the space of orderings.}

It follows from the previous analysis that there are two types of orderings on $S O L$ : those which are Conradian, and those which are induced by affine actions.

- Conradian orderings. These always form a closed subset [15]. Here, Conradian orderings are exactly those for which the normal subgroup $H \simeq \mathbb{Z}^{2}$ is convex. Therefore in $\mathcal{L O}(S O L)$ there are two copies of the Cantor set $\mathcal{L O}\left(\mathbb{Z}^{2}\right)$, each of which corresponding to a choice of sign for $t$. Let us briefly recall the description of the space of left-orderings of $\mathbb{Z}^{2}$. First observe that each oriented line passing through the origin in $\mathbb{R}^{2}$ delimits a unique half-plane (say the one on its right) defining the positive cone of some pseudo-ordering on $\mathbb{Z}^{2}$. The set of elements of $\mathbb{Z}^{2}$ which belong to the line form a cyclic convex subgroup, which is trivial precisely when the pseudo-ordering is an ordering (this happens exactly when the slope of the line is irrational). It follows that this space of pseudo-orderings is naturally parametrized by the unit circle. When the slope is rational, one needs to specify an ordering of the convex subgroup, which is determined by a sign. Therefore the space of orderings of $\mathbb{Z}^{2}$ can be parametrized by a Cantor set obtained by "doubling" each rational point of the circle (see [25]). For simplicity in Figure 2, we ignore this "blow up" procedure and represent each copy of $\mathcal{L O}\left(\mathbb{Z}^{2}\right)$ as a "vertical" circle.

- Bi-orderings. Now observe that among these orderings on $\mathbb{Z}^{2}$, those which are invariant under conjugation by $t$ are precisely those corresponding to lines which are eigendirections of the matrix $T$. The corresponding orderings of $S O L$ are those which are bi-invariant. Taking into account the choices of orientations, this gives precisely eight 6 bi-orderings.

- Affine orderings. The complement of Conradian orderings, namely those induced by affine actions, is an open subset with eight accumulation points, namely $S O L$ 's bi-orderings. We represent these affine orderings by four copies of $\mathbb{R}$, compactified at $\pm \infty$ by pairs of biorderings corresponding to two different eigendirections. This requires some explanation.

First, these four intervals are to be thought as Cantor sets. Similarly to our description of orderings of $\mathbb{Z}^{2}$, one can first consider pseudo-orderings $\preceq_{x}^{(i)}, i=1 \ldots 4$, induced by the orbit of one point, $x$ under an affine action of $S O L$ on the line. Such pseudo-ordering is determined by the following data: first, choose an orientation of the line, then one needs to specify the action of $t$ by multiplication by one of the two eigenvalues of $T$. Hence these pseudo-orderings are naturally parametrized by four copies of the real line. Note that $\preceq_{x}^{(i)}$ is an ordering precisely

\footnotetext{
${ }^{5}$ Recall that a Radon measure is a measure giving finite mass to compact sets.

${ }^{6} \mathrm{~A}$ classification of finitely generated groups admitting only finitely many bi-ordering can be found in [1].
} 
when the stabilizer of $x$ is trivial, which happens on the complement of some dense countable subset $D \subset \mathbb{R}$ (this subset corresponds to the possible values of translations of elements of $\mathbb{Z}^{2}$ ). Otherwise, to define an ordering on $S O L$, one needs to specify an orientation on the convex cyclic subgroup of homotheties fixing $x$. Therefore the subset of affine orderings is locally a Cantor set, obtained by doubling points belonging to $D$ in each of the four copies of $\mathbb{R}$.

Consider one of these intervals, corresponding to an action where $t$ acts by dilation (i.e. $t^{-1}$ acts by contraction), and let $\preceq_{x}^{(1)}$ be the pseudo-ordering associated to the orbit of $x$. Observe that when $x$ goes to $\infty$, the action of $t$ becomes predominant over translations, so that $\preceq_{x}^{(1)}$ converges to an ordering where translations form a convex subgroup. Since the restriction of $\preceq_{x}^{(i)}$ to $H$ is conjugation invariant, one easily checks that this limiting ordering is one of our 8 bi-orderings.

Based on this description, it is not difficult to describe the dynamics of the action of $S O L$ on its space of ordering, see for instance Remark 2.11, We leave this as an exercise to the reader.

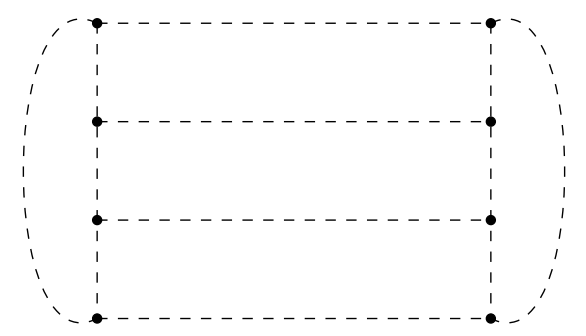

Figure 2: The space of left-orderings of $\mathbb{Z}^{2} \rtimes_{T} \mathbb{Z}$.

\section{Plante's action of $\mathbb{Z} \nmid \mathbb{Z}$.}

The case of $S O L$ which has been described in the previous section is a good representative of what happens for solvable groups with finite rank. The goal of this subsection is to illustrate the difficulties arising when dealing with solvable groups of infinite rank. For simplicity, we shall focus on Abelian-by-cyclic groups $H \rtimes \mathbb{Z}$. The prototypical example of such group is $\Gamma=\mathbb{Z} \geq \mathbb{Z}=\oplus_{i \in \mathbb{Z}} \mathbb{Z} \rtimes$, where $\mathbb{Z}$ acts by shifting the indices in $H=\oplus_{i \in \mathbb{Z}} \mathbb{Z}$.

In the finite rank case, we only have to consider two cases: either $H$ has a global fixed point, or (has an element which) acts freely. Here by contrast, since $H$ is not supposed to have finite rank, a third case can happen for which all elements of $H$ have fixed points, while $H$ does not have any global fixed point7. In [19, Sec. 5], Plante describes an explicit action of $\mathbb{Z} Z \mathbb{Z}$ corresponding to this third case (in particular such an action does not quasi-preserve any Radon measure).

We shall now recall the main properties of Plante's action of $\mathbb{Z} \imath \mathbb{Z}$ which are responsible for the fact that the correspondings induced orderings are non-isolated. Let $t$ be a generator of the cyclic group acting on $H$. For this action, each non-trivial element $h$ of $H$ preserves a compact interval containing 0 in its interior. Let $I_{h}$ be the minimal such interval. These intervals are nested, and their interesection is reduced to $\{0\}$. The element $t$ acts as an expanding homeomorphism having 0 as its unique fixed point, and such that $t\left(I_{h}\right)=I_{t h t^{-1}}$. Somehow, this action reminds of an affine action where $t$ would be some kind of homothety, while elements of $H$ would play the role

\footnotetext{
${ }^{7}$ In the proof of Theorem 1.1 the first one of these three cases (concerning Conradian orderings) is treated implicitely as an initial step in our argument.
} 
of translations. It turns out that similarly to its affine cousin, this ordering can be approximated by its conjugates. Indeed, take for instance the pseudo-ordering induced by the orbit of 0 . The cyclic subgroup generated by $t$ being the minimal convex subgroup, this pseudo-ordering can be completed to an ordering by specifying the sign of $t$. One easily checks that if $t \succ 0$ (resp. $t \prec 0$ ), then for any sequence of points $x_{n}$ converging to zero from the right (resp. left), the sequence of orderings $\preceq_{x_{n}}$ converges to $\preceq$, while being distinct from it.

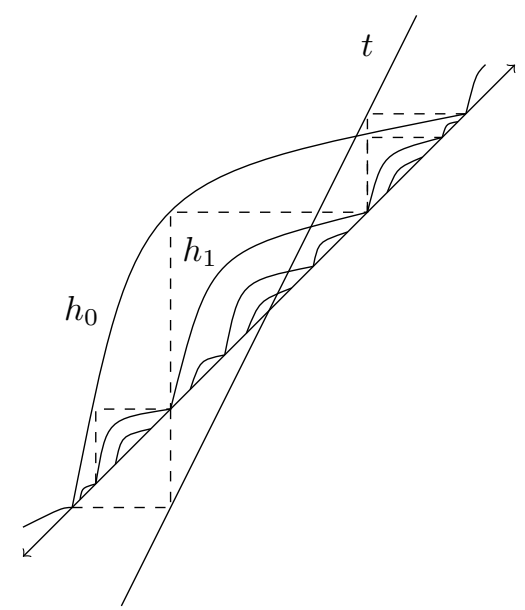

Figure 3: Plante's action: $h_{1}=t h_{0} t^{-1}$.

\section{$5 \quad$ Proof of Theorem 1.1}

Let $\preceq$ be a left-ordering on $\Gamma$. We assume that the convex series of $(\Gamma, \preceq)$ is finite, because otherwise, from Corollary $2.3, \preceq$ is non-isolated. Say the convex series is

$$
\{i d\}=C_{n} \subset C_{n-1} \subset \ldots \subset C_{0}=\Gamma .
$$

We let $T=C_{j+1} \subseteq \Gamma$ be the Conradian soul of $(\Gamma, \preceq)$, which is the maximal convex subgroup on which the restriction of $\preceq$ is Conradian. By [15, 18], if $T$ is not a Tararin group, then $\preceq$ is non-isolated. So we shall assume that $T$ is a Tararin group.

If $T=\Gamma$, then we are done: $\Gamma$ admits only finitely many left-orderings. So we suppose that $T=C_{\ell}$ is a proper convex subgroup of $\Gamma$. We will show that the restriction of $\preceq$ to $C_{\ell-1}$ is nonisolated. Hence, in light of Proposition 2.1, there is no harm in assuming that there are no convex subgroups between $T$ and $\Gamma$.

To show that $\preceq$ is non-isolated, we consider a dynamical realization of $(\Gamma, \preceq)$. We let $I_{T}$ be the minimal interval stable by $T$ and containing 0 . The following is a direct consequence of the fact that $T$ is convex.

Lemma 5.1. Every element of $\Gamma$ either fixes, or moves $I_{T}$ disjointly from itself. In particular, the stabilizer of $I_{T}$ is exactly $T$.

We let $\widetilde{\Gamma}$ be a finite index, normal, solvable subgroup of $\Gamma$. We let $\widetilde{\Gamma}^{0}=\widetilde{\Gamma}$ and $\widetilde{\Gamma}^{i}=\left[\widetilde{\Gamma}^{i-1}, \widetilde{\Gamma}^{i-1}\right]$ be the associated derived series

$$
\{i d\}=\widetilde{\Gamma}^{k} \triangleleft \widetilde{\Gamma}^{k-1} \triangleleft \ldots \triangleleft \widetilde{\Gamma}^{1} \triangleleft \widetilde{\Gamma} \triangleleft \Gamma .
$$

Note that each $\widetilde{\Gamma}^{i}$ is normal in $\Gamma$. We fix once and for all $i$, being the minimal index such that $\widetilde{\Gamma}^{i} \subseteq T$. Since $T$ is a proper convex subgroup, we have that $k \geq i \geq 1$. In a diagram 


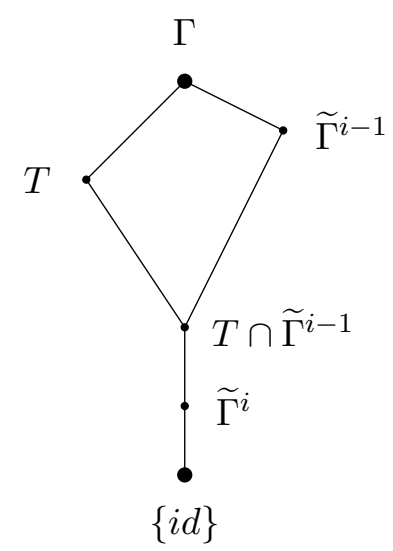

Lemma 5.2. The order restricted to $\widetilde{\Gamma}^{i-1}$ is Conradian.

Proof: Indeed, $\widetilde{\Gamma}^{i-1} \cap T$ is convex and normal in $\widetilde{\Gamma}^{i-1}$. Moreover, its quotient is Abelian, so it admits only Conradian orderings. The lemma then follows from Remark 2.2.

Lemma 5.3. The orbit of 0 under $\widetilde{\Gamma}^{i-1}$ accumulates on $\pm \infty$.

Proof: Let $I$ be the smallest open interval containing 0 , and stable under $\widetilde{\Gamma}^{i-1}$. Since $\widetilde{\Gamma}^{i-1}$ is normal in $\Gamma, I$ is either fixed or moved disjointly by any $\gamma \in \Gamma$. In particular, $\operatorname{Stab}_{\Gamma}(I)$, the stabilizer of $I$, is a convex subgroup. Now, if $I \neq \mathbb{R}$, then the maximality of $T$ implies $\operatorname{Stab}_{\Gamma}(I) \subseteq T$. But this implies $\widetilde{\Gamma}^{i-1} \subset T$, which is contrary to our assumptions.

We have two cases to analyze in order to prove Theorem 1.1.

- Case 1. Suppose there is $g_{0} \in \widetilde{\Gamma}^{i-1}$ having no fixed points. Such case occurs for instance if $\widetilde{\Gamma}^{i-1}$ has finite rank. Combining the results from [15, 19] we obtain the following proposition (by measure we shall implicitely mean a Radon measure which is finite on compact sets).

Proposition 5.4. The action of $\Gamma$ on the real line is semi-conjugated to a non-Abelian affine action $\varphi: \Gamma \rightarrow$ Af $f_{+}(\mathbb{R})$.

Proof: Since the action of $\widetilde{\Gamma}^{i-1}$ is Conradian and there is $g_{0} \in \widetilde{\Gamma}^{i-1}$ without fixed points, Corollary 2.15 ensures that $\widetilde{\Gamma}^{i-1}$ has a maximal proper convex subgroup $N \supseteq \widetilde{\Gamma}^{i}$, which is normal. In particular, $N$ fixes some open bounded interval $I_{N}$ around 0 . The action of $\widetilde{\Gamma}^{i-1}$ is semi-conjugated to an action factoring through $A=\widetilde{\Gamma}^{i-1} / N$. Since $A$ is Abelian and has an element acting without fixed point, [19, Proposition 3.1] implies that the corresponding action has an invariant measure. Lifting back this measure yields a measure $\mu$ which is preserved by $\widetilde{\Gamma}^{i-1}$ in the original action.

Since $g_{0}$ acts without fixed points, the translation number homomorphism $\tau_{\mu}: \widetilde{\Gamma}^{i-1} \rightarrow \mathbb{R}$, given by $\tau_{\mu}(g)=\mu((0, g(0)])$, is non trivial (here and below, we use the convention $\mu([x, y])=$ $-\mu([y, x])$ for $y<x)$. It now follows from [19, Lemma 4.2 and 4.3] that there is a measure, which we still call $\mu$, which is quasi-preserved by $\Gamma$, meaning that for every $\gamma \in \Gamma$, there is a positive real number $\lambda_{\gamma}$ such that $\gamma_{*}(\mu)=\lambda_{\gamma} \mu\left(\right.$ where $\left.\gamma_{*}(\mu)(X):=\mu\left(\gamma^{-1}(X)\right), X \subseteq \mathbb{R}\right)$. In this way we have a homomorphism $\varphi: \Gamma \rightarrow A f f_{+}(\mathbb{R})$, which extends $\tau_{\mu}$, given by

$$
\varphi(\gamma)(x)=\frac{1}{\lambda_{\gamma}} x+\mu((0, \gamma(0)])
$$


This affine action is semi-conjugated to the original dynamical realization action of $(\Gamma, \preceq)$. Indeed, if for $x \in \mathbb{R}$ we let $F(x)=\mu((0, x])$, then a direct computation shows that

$$
F(\gamma(x))=\varphi(\gamma)(F(x))
$$

It only remains to check that $\varphi(\Gamma)$ is non-Abelian. To this end we let $I_{\mu}:=(a, b)$, where $a=\sup \{x<0 \mid x \in \operatorname{supp}(\mu)\}$, and $b=\inf =\{x>0 \mid x \in \operatorname{supp}(\mu)\}$. Then, since $\mu$ is quasi-preserved (so in particular its support $\operatorname{supp}(\mu)$ is preserved) we have that $\operatorname{Stab}_{\Gamma}\left(I_{\mu}\right)$ is either fixed or moved disjointly. In particular, $\operatorname{Stab}_{\Gamma}\left(I_{\mu}\right)$ is a proper convex subgroup. So, $\operatorname{Ker}(\varphi) \subseteq \operatorname{Stab}_{\Gamma}\left(I_{\mu}\right) \subseteq T$. Therefore if the affine action of $\Gamma$ was Abelian, then $T$ would be a normal and co-Abelian subgroup, so Remark 2.2 would imply that $\preceq$ is Conradian, which is contrary to our assumptions.

Lemma 5.5. The kernel of $\varphi$ is a convex subgroup of $(\Gamma, \preceq)$.

Proof: We keep the notations of the proof of Proposition [5.4,

We first claim that $\operatorname{Stab}_{\Gamma}\left(I_{\mu}\right)=T$ (equivalently $I_{\mu}=I_{T}$ ). Indeed, let $\preceq^{\prime}$ be the pseudoordering of $\Gamma$ induced by $\varphi$ at $F(0)=F\left(I_{\mu}\right)$. Since $\varphi$ is an affine action, $\preceq^{\prime}$ has only one convex subgroup, namely $\{\gamma \in \Gamma \mid \varphi(\gamma)(F(0))=F(0)\}=\operatorname{Stab}_{\Gamma}\left(I_{\mu}\right)$. However, equation (2) implies that $\preceq^{\prime}$ is the quotient of $\preceq$ by $\operatorname{Stab}_{\Gamma}\left(I_{\mu}\right): \varphi(g) \succ^{\prime} i d \Rightarrow g \succ i d$. Thus, the claim follows from Remark 2.5.

We now show that $\operatorname{Ker}(\varphi)$ is convex in $(\Gamma, \preceq)$. First observe that the previous claim implies that $\varphi(T)$ is the Abelian subgroup of homotheties centered at $F(0)$. If it was trivial, then we would have that $T=\operatorname{ker} \varphi$ is convex. Let us therefore suppose that $\varphi(T)$ is non-trivial.

We let $\{i d\}=T_{0} \triangleleft T_{1} \triangleleft \ldots \triangleleft T_{m}=T$ be the convex series of the Tararin group $T$. Recall that $T_{i} / T_{i-1}$ has rank 1 and that the action of $T_{i+1}$ on $T_{i} / T_{i-1}$ is by multiplication by some negative number. In particular $T / T_{m-1}$ is the unique torsion-free Abelian quotient of $T$. It follows that $T / T_{m-1}=\varphi(T)$ and $\operatorname{ker} \varphi=T_{m-1}$ is convex.

Now the proof of Theorem 1.1 in case 1 follows from Corollary 2.13 .

- Case 2. Suppose every element $g \in \widetilde{\Gamma}^{i-1}$ has a bounded domain (i.e. an open interval fixed by $g$ with no fixed point of $g$ in its interior) $I_{g}$ around 0.

Our proof of Theorem 1.1 in Case 2 consists in showing that $\preceq$ can be approximated by a left-ordering induced from the dynamical realization of $(\Gamma, \preceq)$, where the first reference point is chosen outside but very close to $I_{T}$. For this purpose, we shall prove that the action is quite similar to the one described in $\$$ (except that there, $I_{T}$ was reduced to a point).

It follows from Lemmas 5.2 and 5.3 that the union of the $I_{g}$ is all of $\mathbb{R}$. In the sequel, we exploit the facts that $\widetilde{\Gamma}^{i-1}$ is normal in $\Gamma$ and that the order restricted to $\widetilde{\Gamma}^{i-1}$ is Conradian to give a more precise picture of the action. First, Corollary 2.16 immediately implies

Lemma 5.6. Let $f \in \Gamma$ and $g \in \widetilde{\Gamma}^{i-1}$. Then one, and only one of the following happen

$-f\left(I_{g}\right)=I_{g}$ or

- $f\left(I_{g}\right)$ is disjoint from $I_{g}$ or either

- (up to changing $f$ by its inverse) $\overline{I_{g}} \subset f\left(I_{g}\right)$, i.e. $f$ acts as dilation on $I_{g}$.

The following corollaries are easy and left to the reader. 
Corollary 5.7. Let $g \in \widetilde{\Gamma}^{i-1} \backslash T$, and $I_{g}$ its domain. If $t \in T$, then $t$ either fixes $I_{g}$ or acts on it as a dilation.

Corollary 5.8. If $I$ is an interval obtained as a union or an intersection of $I_{g}$ 's for $g \in \widetilde{\Gamma}^{i-1}$, then a weak form of Lemma [5.6 holds for I. Namely every element $f \in \Gamma$ either moves I disjointly from itself, or up to replacing $f$ by its inverse, $I \subset f(I)$ (if the intersection is strict, we say that $f$ weakly dilates $I$ ).

Using the fact that Tararin groups have finite rank, we now obtain a useful description of $I_{T}$..

Lemma 5.9. The intersection of $I_{g}$ for $g \in \widetilde{\Gamma}^{i-1} \backslash T$ coincides with $I_{T}$.

Proof: First observe that Theorem 2.14 implies that the $I_{g}$ 's, for $g \in \widetilde{\Gamma}^{i-1} \backslash T$ are totally ordered for the inclusion. On the other hand, by Corollary [5.8, if no element weakly dilates $J$, then the stabilizer of $J$ is convex. Thus it contains $T$ by Corollary 5.7 (which also applies to $J$ ), so it must be equal to $T$. But this implies that $J=I_{T}$.

It is therefore enough to prove that no element weakly dilates $J$. Suppose by contradiction that $f^{-1}$ weakly dilates $J$ (i.e. $f$ weakly contracts $J$ ). Then there exists $g \in \widetilde{\Gamma}^{i-1} \backslash T$ such that $J \cap I_{g^{f}}$ is strictly contained in $J$, hence that $g^{f}$, and more generally $g_{n}=g^{f^{n}}$ belongs to $T$ for all $n \geq 1$. Moreover, $f^{n}\left(I_{g}\right)$ is a minimal interval (not necessarily containing 0 ) fixed by the element $g_{n}$. Since $f$ acts as a dilation on $I_{g}$, we deduce that $f^{n+1}\left(I_{g}\right) \subsetneq f^{n}\left(I_{g}\right)$. In particular $g_{k} \in S_{n} \forall k \geq n$, where $S_{n}=\operatorname{Stab}_{T}\left(f^{n}\left(I_{g}\right)\right)$. Let $x$ be a point in the decreasing intersection of compact intervals $\overline{f^{n}\left(I_{g}\right)}$. We have that $\left(S_{n}\right)_{n \geq 1}$ is a strictly decreasing sequence of convex subgroups of $T$ for the pseudo-ordering $\preceq_{x}$, violating the fact that $T$ has only finitely many orderings (and more generally one could check that this is incompatible with the fact that $T$ has finite rank).

Since there are no proper convex subgroups above $T$, we have

Lemma 5.10. For any $g \in \widetilde{\Gamma}^{i-1} \backslash T$, there exists $f \in \Gamma$ acting as a dilation on $I_{g}$. Moreover, for every $g, g^{\prime} \in \widetilde{\Gamma}^{i-1} \backslash T$ satisfying $I_{g} \subseteq I_{g^{\prime}}$, there exists $f \in \Gamma$ such that $I_{g^{\prime}} \subseteq f\left(I_{g}\right)$.

Proof: Looking for a contradiction, suppose that there exists $g \in \widetilde{\Gamma}^{i-1} \backslash T$ such that elements of $\Gamma$ either stablize $I_{g}$ or move it disjointly from itself. It follows that the stabilizer $S$ of $I_{g}$ is convex. By Corollary [5.7, $S$ contains $T$, and the inclusion is strict as $S$ contains $g$. On the other hand $S$ cannot be all of $\Gamma$ since $I_{g}$ is bounded: a contradiction. This shows the first part of the lemma.

Let us prove the second statement of the lemma. Let $\mathcal{F}=\left\{f \in \Gamma \mid f\right.$ dilates $\left.I_{g}\right\}$. First note that for all $f \in \mathcal{F}$, we have $f\left(I_{g}\right)=I_{g^{f}}$. Therefore the set of all $f\left(I_{g}\right)$ for $f \in \mathcal{F}$ is nested, let $I$ be its union. We claim that $I$ is either fixed or moved disjointly.

Indeed, let us first suppose that there exists $h \in \widetilde{\Gamma}^{i-1}$ such that $h(I)$ strictly contains $I$. A continuity argument implies that there exits $f \in \mathcal{F}$ such that $h \circ f\left(I_{g}\right)$ contains $f\left(I_{g}\right)$ (hence $I_{g}$ ) and is not contained in $I$. The first of these statements implies that $h \circ f$ belongs to $\mathcal{F}$, while the other implies that it does not (by definition of $I$ ), so this case cannot occur. Hence, from Corollary [5.8, our claim follows. In particular, the stabilizer of $I$ is a convex subgroup containing $T$, so $I$ must be all of $\mathbb{R}$. Hence the lemma.

We have given a combinatorial description of the dynamics of $\Gamma$ around $I_{T}$. We now exploit this description to approximate $\preceq$. First we show that orderings induced by points outside of $I_{T}$ are distinct from $\preceq$. 
Lemma 5.11. For all $x$ not in $\overline{I_{T}}$, there exists $f \in \Gamma$ such that $f \prec_{x} 0$ and $f \succ 0$. In particular, any left-ordering induced from the dynamical realization of $(\Gamma, \preceq)$ with $x$ as first reference point is different from $\preceq$.

Proof: Let $x \notin \overline{I_{T}}$, say on its left (the other case is symmetric). It results from Lemma 5.9 that one can find $g, g^{\prime} \in \widetilde{\Gamma}^{i-1} \backslash T$ such that $I_{g} \subset I_{g^{\prime}}$ and $x \in I_{g^{\prime}} \backslash I_{g}$. On the other hand, Lemma 5.10 provides us with an element $f$ such that $f\left(I_{g}\right)$ contains $I_{g^{\prime}}$. In particular, if $f \succ i d$, then we are done because $f \prec_{x} i d$. So we assume that $f \prec i d$. Up to changing $g$ by $g^{-1}$, we can assume that $g \succ i d$. In particular, $g\left(I_{T}\right)$ is moved to the right of $I_{T}$. This implies, that for $n$ large enough, $g^{-n} f g^{n} \succ i d$. But, in the same time, $g^{-n} f g^{n}\left(I_{g}\right)=g^{-n} f\left(I_{g}\right)=g^{-n}\left(I_{g^{f}}\right)=f\left(I_{g}\right)$, where the last equality follows because $g$ and $g^{f}$ are not crossed and $I_{g} \subset I_{g^{f}}$. Hence the lemma.

The following step consists in showing that when $x_{n}$ converges to an end point of the interval $I_{T}$, then $\preceq_{x_{n}}$ converges to $\preceq$ outside of $T$.

Lemma 5.12. If $x_{n}$ converges to an end point $x$ of $I_{T}$, then $\preceq_{x_{n}}$ converges to $\preceq$ outside of T. More precisely, for every $g \in \Gamma \backslash T$, we have that $g \succ 0$ if and only if $g \succ_{x_{n}} 0$ for all $n$ large enough.

Proof: Since the stabilizer of $I_{T}$ is precisely $T$, given any $\preceq$-positive $g \in \Gamma \backslash T$, we have that $g$ moves $I_{T}$ disjointly to its right. Therefore $g \succ 0$ if and only if $g \succ_{x} 0$. The lemma then follows by continuity.

To prove Theorem [1.1, we are left to proving that for a well-chosen sequence $\left(x_{n}\right)$ converging to an end point of $I_{T}$, the orderings $\preceq_{x_{n}}$ converges to $\preceq$ in restriction to $T$. We shall use in a crucial way the fact that Tararin groups have "very few" actions on the real line. More precisely, we have two following lemmas where the action considered is still the dynamical realization of $(\Gamma, \preceq)$. Recall that the convex series of $T$ is always given by $\{i d\}=T_{0} \triangleleft T_{1} \triangleleft$ $\ldots \triangleleft T_{m-1} \triangleleft T_{m}=T$. We let $\gamma_{T} \in T$ be a non trivial element in $T / T_{m-1}$ which acts on $T_{m-1} / T_{m-2}$ by a multiplication by a negative (rational) number.

Lemma 5.13. If $\gamma_{T}$ fixes some point $x$, then so does $T$.

Proof: Suppose $x$ is fixed by $\gamma_{T}$ but not by $T$. We can then induce a left-ordering on $T$ with reference point $\left(x, x_{2}, \ldots\right)$. In this ordering the stabilizer of $x$ is a proper convex subgroup, which is impossible since it does not coincide with any of the subgroups $T_{i}$.

Lemma 5.14. For any $y \in \mathbb{R}$, which is not fixed by $\gamma_{T}$, there is $x$ between $\gamma_{T}^{-2}(y)$ and $\gamma_{T}^{2}(y)$ such that $\preceq$ and $\preceq_{x}$ coincide over $T_{m-1}$.

Proof: Recall that in a dynamical realization, the set of fixed points of a non-trivial element has empty interior. Since $T$ is countable, there is a point $z$ between $y$ and $\gamma_{T}^{-1}(y)$ whose orbit under $T$ is free. In particular $\preceq_{z}$ is a total ordering of $T$. Since $T_{m-1}$ is convex in $\preceq_{z}$, there is a minimal open interval $I$ containing $z$ and which is stabilized by $T_{m-1}$. Being moved disjointly from itself by any non-trivial power of $\gamma_{T}, I$ contains at most one point of the orbit of $y$ under $\left\langle\gamma_{T}\right\rangle$. In particular, $I$ is (strictly) contained between $\gamma_{T}^{-2}(y)$ and $\gamma_{T}(y)$. Now by Proposition 2.10, there exists an element $g$ either in $T_{m-1}$ or in $\gamma_{T} T_{m-1}$ such that $\preceq$ and $g\left(\preceq_{z}\right)=\preceq_{g z}$ coincide over $T_{m-1}$. Clearly $x=g z$ satisfies the conclusion of the lemma.

The last step in the proof of Theorem 1.1 is achieved by the following lemma. 
Lemma 5.15. There exists a sequence $\left(x_{n}\right)$ converging to an end point of $I_{T}$ from outside such that the induced left-ordering $\preceq_{x_{n}, 0}$ coincides with $\preceq$ over $T$ for all $n$.

Indeed, combining Lemmas 5.12 and 5.15 , We have that $\preceq_{x_{n}, 0}$ converges to $\preceq$. On the other hand, Lemma 5.11 shows that $\preceq_{x_{n}, 0}$ and $\preceq$ are different. This shows Theorem 1.1 in Case 2 .

Proof of Lemma 5.15: The idea is to take $x \notin \overline{I_{T}}, x$ close to $\partial I_{T}$, such that the sign of $\gamma_{T}$ is preserved and then to use Lemmas 5.13 and 5.14

By Lemma 5.9, there exists a sequence $g_{n} \in \widetilde{\Gamma}^{i-1} \backslash T$ such that $I_{g_{n}}$ converges to $I_{T}$.

- Subcase 1. $\gamma_{T}$ dilates $I_{g_{n}}$.

Up to taking a subsequence, we can assume that $I_{g_{n}} \subset I_{g_{n-1}}$. For concreteness we suppose $\gamma_{T} \prec i d$, and let $y_{n}$ be the left end-point of $I_{g_{n}}$, so that the sequence $\left(y_{n}\right)$ converges to the left end-point $z$ of $I_{T}$ (in the case $\gamma_{T} \succ i d$, we take $y_{n}$ being right endpoints). Since $\gamma_{T}$ dilates $I_{g_{n}}, \gamma_{T} \prec y_{n} i d$. Let $x_{n}$ be the sequence of points obtained from Lemma 5.14 between $\gamma_{T}^{-2}\left(y_{n}\right)$ and $\gamma_{T}^{2}\left(y_{n}\right)$ such that $\preceq$ and $\preceq_{x_{n}}$ coincide in restriction to $T_{m-1}$. By continuity of $\gamma_{T}$ and its inverse, the sequence $\left(x_{n}\right)$ converges to the same limit $z$. This shows the lemma in Subcase 1.

- Subcase 2. $\gamma_{T}$ (hence $T$ ) fixes $I_{g_{n}}$.

Again we let $x_{n}$ be the left end-point of $I_{g_{n}}$. Observe that $\preceq_{x_{n}}$ is a pseudo-ordering on $\Gamma$, for which the stabilizer $S_{n}$ of $x_{n}$ is obviously convex and, from Lemma 5.13, contains $T$. In particular, the left-ordering $\preceq_{x_{n}, 0}$ coincides with $\preceq$ over $T$. This ends the proof of Lemma 5.15 .

\section{References}

[1] R. Botto-Mura \& A. Rhemtulla. Orderable groups. Lecture notes in Pura and Applied Mathematics, Vol 27. Marcel Dekker, New York (1977).

[2] I. M. Chiswell And P. H. Kropholler, Soluble right orderable groups are locally indicable, Canad. Math. Bull. 36 (1993), 22-29.

[3] P. Conrad. Right-ordered groups. Mich. Math. Journal 6 (1959), 267-275.

[4] P. Dehornoy. Monoids of $\mathcal{O}$-type, subword reversing, and ordered groups Preprint, arxiv 1204.3211.

[5] B. Deroin.. The group of almost-periodic homeomorphisms of the real line. Enseignement Mathématique. To appear.

[6] T. V. Dubrovina \& N. I. Dubrovin. On Braid groups, Mat. Sb. 192 (2001), 693-703.

[7] É. GHYs. Groups acting on the circle, Enseign. Math. 47 (2001), 329-407.

[8] T. Iто. Dehornoy-like left orderings and isolated left orderings J. of Algebra 374 (2013), 42-58.

[9] T. Iто. Constructions of isolated left-orderings via partially central cyclic amalgamation, Preprint, arxiv 1107.0545 .

[10] V. Kopytov \& N. Medvedev. Right ordered groups. Siberian School of Algebra and Logic, Plenum Publ. Corp., New York (1996).

[11] P. Linnell. The space of left orders of a group is either finite or uncountable. London Math. Soc. 43 (2011), 200-202. 
[12] S.H. MCClEARY. Free lattice-ordered group represented as o-2 transitive l-permutation groups. Trans. Amer. Math. Soc., 290(1) (1985), 69-79.

[13] D. W. Morris. Amenable groups that act on the line. Algebraic \& Geometric Topology 6 (2006), 2509-2518.

[14] R. B. Mura And A. H. Rhemtulla, Orderable groups, Lecture Notes in Pure and Appl. Math., vol. 27, Marcel Dekker, New York (1977).

[15] A. Navas. On the dynamics of (left) orderable groups. Ann. Inst. Fourier (Grenoble) 60 (2010), 1685-1740.

[16] A. Navas. A remarkable family of left-ordered groups: central extensions of Hecke groups. J. of Algebra 328 (2011), 31-42.

[17] A. Navas. Groups of circle diffeomorphisms. Chicago lect. in Math (2011). Spanish version published in Ensaios Matemáticos, Braz. Math. Soc. (2007).

[18] A. Navas \& C. Rivas, with an Appendix by A. Clay. A new characterization of Conrad's property for group orderings, with applications. Algebraic \& Geometric Topology 9 (2009), 20792100.

[19] J.F. Plante. On solvable groups acting on the real line. Trans. Amer. Math. Soc. 278 (1983), 401-414.

[20] M. Raghunathan. Discrete subgroups of Lie groups. Springer-Verlag (1972).

[21] C. Rivas. On the spaces of Conradian group orderings. Journal of group theory 13 (2010), 337-353.

[22] C. Rivas. On groups with finitely many Conradian orderings. Comm. in Alg. 40 (2012), 25962612.

[23] C. Rivas. Left-orderings on free products of groups. J. of Algebra 350 (2012), 318-329.

[24] D.J.S. Robinson Finiteness condition and generalized solvable groups 1-2. Springer-Verlag (1972).

[25] A. Sikora. Topology on the spaces of orderings of groups. Bull. London Math. Soc. 36 (2004), 519-526.

Cristóbal Rivas

UMPA, ENS-Lyon

Email: cristobal.rivas@ens-lyon.fr

Romain Tessra

UMPA, ENS-Lyon

Email: Romain.Tessera@ens-lyon.fr 